\title{
A concept of software extension of 3D low-PRF radar systems to 4D semi-medium-PRF radar systems
}

\section{Michał Meller, Kamil Stawiarski}

Michał Meller, Kamil Stawiarski, "A concept of software extension of 3D lowPRF radar systems to 4D semi-medium-PRF radar systems," Proc. SPIE 11442, Radioelectronic Systems Conference 2019, 1144203 (11 February 2020); doi: $10.1117 / 12.2564904$

SPIE. Event: Radioelectronic Systems Conference 2019, 2019, Jachranka, Poland 


\title{
A Concept of Software Extension of 3D Low-PRF Radar Systems to 4D Semi-Medium-PRF Radar Systems
}

\author{
Michał Meller *a,b, Kamil Stawiarski a,b \\ ${ }^{\text {a) PIT-RADWAR S.A., Poligonowa 30, 04-051 Warsaw, POLAND }}{ }^{1}$ \\ b) Gdańsk University of Technology, \\ Faculty of Electronics, Telecommunications and Computer Science \\ Department of Automatic Control, Narutowicza 11/12, 80-233 Gdańsk, POLAND
}

\begin{abstract}
We present a concept of software modification of three-dimensional (3D) radar systems, designed to work in the low pulse repetition frequency mode, that equips them with the ability to estimate the radial velocity and to properly measure the range of targets that are detected outside the radar's instrumented range. Despite the fact that the proposed modifications are designed so as to require only minor changes in software, they offer significant growth in the system capabilities. The modified system may potentially work in the medium pulse repetition frequency mode as a so-called four-dimensional (4D) system. The proposed Doppler velocity estimation algorithm is presented in details as well.
\end{abstract}

Keywords: radar, range-Doppler ambiguity, estimation.

\section{INTRODUCTION}

The two primary roles of radar systems are to detect targets of interest in the surrounding space and to determine their coordinates. Depending on the number of estimated target coordinates, radars are typically characterized as either twodimensional (2D) or three-dimensional (3D) systems. While 2D radars are capable of estimating only the range and the azimuth of the detected targets, 3D systems can additionally determine the targets' elevation angle, or height. The overwhelming majority of modern military radars belong to the class of 3D systems, which stems from the value of the height information in, among others, decision-making processes or fire control.

In addition to determining the targets' range, azimuth, and elevation coordinates, by exploiting the Doppler effect, radars can also measure the radial velocity from a single target illumination. Three-dimensional systems equipped with this capability are sometimes referred to as 4D systems. Ground-based 4D surveillance systems do not seem particularly widespread, because the full velocity vector is typically worked out by the radar tracker, which analyzes the movement of the target between its consecutive illuminations. In comparison with the tracker-based approach, the process of estimating the radial velocity from a single illumination is considerably more difficult. The main source of the difficulties is the fact that ground-based surveillance radars often employ the so-called low pulse repetition frequency (PRF), which means that the system PRF is chosen such that the instrumented range of the radar exceeds the maximum detection range of the class of targets the radar was designed to detect. The resultant pulse repetition period (PRP) is typically so large that it may be impossible to determine the radial velocity unambigously by analyzing echo of a single pulse trail because of the, well known in the digital signal processing, aliasing phenomenon - the discrete-time domain Doppler frequency is an alias of its actual, continous-time, value. In this situation, to determine the true value of the Doppler shift it is necessary to illuminate the target with at least two pulse trails that differ in their PRF and to analyze the Doppler spectra of their echos jointly. Note that, even though the tracker-based estimation of velocity is considerably more straightforward, the direct estimation of the „fourth" coordinate has several advantages, such as improved plot-totrack association or better discrimination of false echos, among others. These importance of these advantages on the modern battlefield increases steadily, which makes the direct estimation capability highly desirable

* Corresponding author e-mail: michal.meller@pitradwar.com

Radioelectronic Systems Conference 2019, edited by Piotr Kaniewski, Jan Matuszewski, Proc. of SPIE Vol. 11442, $1144203 \cdot$ (c) 2020 SPIE · CCC code: 0277-786X/20/\$21 · doi: 10.1117/12.2564904 
Another area of improvement for low-PRF systems is the way they handle detections of targets that are outside their instrumented range. Regardless of the fact that low-PRF radar design aims to ensure that targets are detected within the system's instrumented range, targets that are well outside the instrumented range can also be detected occasionally. Without going into unnecessary details, the possibility of such an event stems from the fact that the design of the radar power budget and instrumented range often assumes a specific class of targets, typically characterized by specifying its radar cross section (RCS). In reality, however, the radar may face much broader variety of targets, which may include targets whose RCS is considerably higher than the assumed one. Such targets will be detected outside the system's instrumented range. For example, in case of a radar designed to detect small UAVs (RCS $<0.1 \mathrm{~m}^{2}$ ), the detection range of large aircrafts (RCS $>10 \mathrm{~m}^{2}$ ) may exceed the "nominal" detection range more than threefold.

The detections that originate from targets outside the instrumented range are often treated as undesired, because they are assigned with invalid range, in which case one may seek to eliminate them from appearing. Taking into account, however, that such detections represent actual targets, and that the modern battlefield becomes increasingly net-centric, it seems more and more reasonable to equip radars with mechanisms that allow them to determine the range of such detections properly.

In this paper, we propose the concept of low-cost software modification that equips 3D low-PRF radars with the two capabilities discussed above, namely the capability to estimate the radial velocity from a single illumination, and the capability to resolve the range ambiguity problem for targets outside the instrumented range. As a result, one obtains a 4D system that can potentially work in the medium-PRF mode, which can improve its performance characteristics, such as the detection range or the track accuracy. The proposed radial velocity estimation algorithm is presented in details, while the range estimation algorithm is presented in the sibling paper [2] due to the manuscript length limitations.

The paper is organized in the following way: in Section 2, the proposed solution is presented on a general level. In Section 3, the proposed velocity estimation algorithm is presented. Section 4 presents results of computer simulations. Finally, Section 5 concludes.

\section{CONCEPT OF PROPOSED SOFTWARE MODIFICATION}

The proposed extension can be implemented in coherent radar systems employing the moving target detection (MTD) processing [1,3]. Such radars typically alternate between transmitting two or more pulse trails that differ in their PRF. The purpose of varying the PRF is the elimination of the so-called blind velocities. The blind velocities are such radial velocities that result in the echo of a moving target appearing in the same Doppler filter as the echos of stationary targets or clutter. Denote by $v$ the radial velocity of a target, and let $T_{P}$ be the pulse repetition period. The Doppler shift of the target echo is given by

$$
f_{D}=\frac{2 v}{c} F_{0}
$$

where $c$ is the speed of light and $F_{0}$ is the radar carrier frequency. Equation (1) represents the Doppler shift in the socalled fast-time domain. In the slow-time domain (discrete time enumerated by consecutive transmitted pulses), $f_{D}$ becomes aliased to the value[1,3]

$$
f_{A}=f_{D}-n \frac{1}{T_{P}}
$$

where $n$ is an integer, chosen such that $f_{A} \in\left[-\frac{1}{2 T_{P}}, \frac{1}{2 T_{P}}\right)$. The blind velocity is such velocity that results in $f_{A}=0$, which leads to

$$
v_{B}=n \frac{c}{2 T_{P} F_{0}}
$$

where $\frac{c}{2 T_{P} F_{0}}$ is often referred to as the first blind velocity.

The first blind velocity is typically quite low. For instance, consider a radar operating in the $\mathrm{X}$ band $\left(F_{0}=10 \mathrm{GHz}\right)$ with $T_{P, 1}=500 \mu \mathrm{s}$. Its first blind speed is $v_{B, 1}=30 \mathrm{~m} / \mathrm{s}$, or $108 \mathrm{~km} / \mathrm{h}$. Such radar could therefore have considerable difficulties detecting targets with velocities close to $108 \mathrm{~km} / \mathrm{h}, 216 \mathrm{~km} / \mathrm{h}, 324 \mathrm{~km} / \mathrm{h}$, etc. To prevent this problem, the 
radar might transmit another pulse trail with different PRP, e.g. $T_{P, 2}=550 \mu \mathrm{s}$, which results in the blind velocity of $v_{B, 2}=27.27 \mathrm{~m} / \mathrm{s}$, or $98.18 \mathrm{~km} / \mathrm{h}$. In this situation a target with radial velocity of, for example, $324 \mathrm{~km} / \mathrm{h}$, will be masked by clutter for the pulse trail with PRP equal to $T_{P, 1}$, but it will appear in a clutter-free filter for the segment with PRP $T_{P, 2}$. One can therefore conclude that altering the PRF increase the blind velocity of the radar to the least common multiple of the partial blind velocities $v_{B, 1}$ i $v_{B, 2}$, i.e., to $1080 \mathrm{~km} / \mathrm{h}$.

Apart from increasing the system blind velocity, employing multiple PRFs also allows one to estimate the true radial velocity of targets (as long as it is limited to \pm half of the radar blind velocity), and to estimate the true range of detections that originated from targets outside the radar's instrumented range. In the first case, one should find such velocity that results in the slow-time Doppler shifts (2) that match the actual observations, up to the influence of noise and interferences. In the case of targets outside the instrumented range, varying the PRP changes their apparent (ambiguous) detection range. Therefore, one may resolve the true distance to the target by analyzing the behavior of range estimates for different values of PRP [1].

In case of relatively modern systems, implementing both extensions does not require one to introduce any significant modifications to the radar itself - all that is required is a relatively minor modification of the software. The radar software is typically divided into several functional modules that perform separate tasks, such as the pulse compression, beamforming, Doppler filtering, detection, or the estimation of target coordinates. The last two modules from the above list, i.e., the detection module and the estimation module, are of particular relevance to our considerations.

The role of the detection module is to detect targets. To this end, this piece of software implements algorithms such as the clutter map, the constant false alarm rate (CFAR) detector, the incoherent integration and the thresholding. The output of the module are the lists of the raw detections with added complimentary data in the form of, e.g., the index of the range cell in which the detection occurred and the range-gated echo signals, possibly from several consecutive pulse trails. These signals undergo further processing in the estimation module, which works out the target's azimuth and elevation coordinates.

(a)

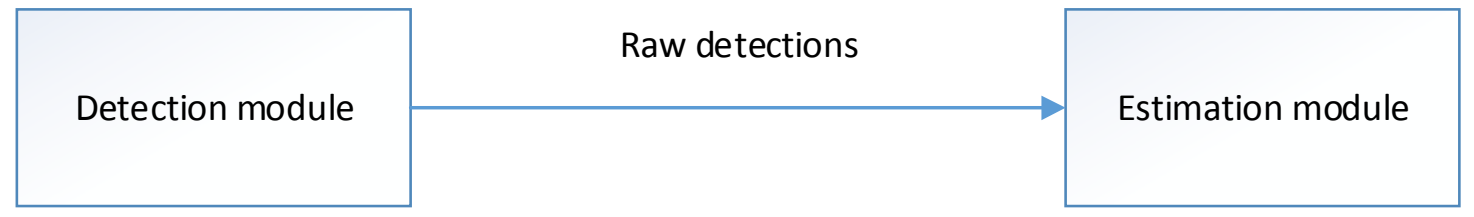

(b)

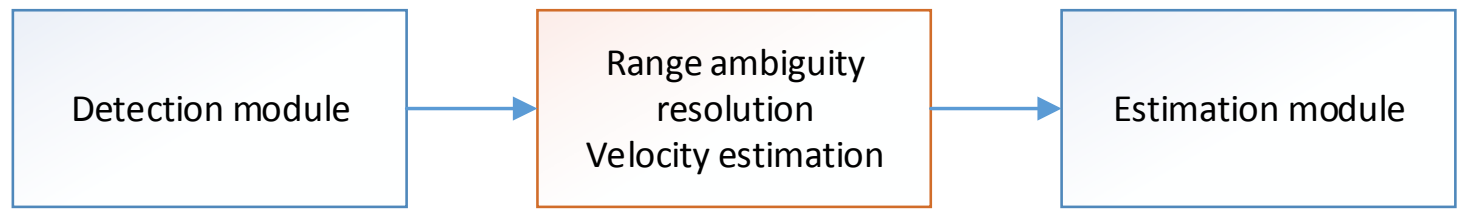

Fig. 1. Block diagram of the proposed software modification: (a) Existing software structure. (b) Proposed software structure.

Even though extending the detection module with a dedicated channel, responsible for detecting targets outside the instrumented range, seems to be the best option in purely technical terms, in the proposed modification only minimal changes in the software are assumed. This is why we propose to leave the existing software "as is", and add an additional functional module between the detection and the estimation modules (see Fig. 1). This new module captures the raw detections, analyzes their contents for the presence of detections from targets outside the instrumented range, and generates a new list with corrected values of range [2]. Moreover, by analyzing the Doppler spectra of the signals that accompany the detections, the module estimates the targets' radial velocities and extends the output list of detections with this information. The modified detections are then forwarded to the estimation module, where - without any 
changes in the software - the azimuth and elevation coordinates are worked out. Note that although the proposed approach is not optimal, it can be implemented with minimal effort, which has its advantages.

\section{RADIAL VELOCITY ESTIMATION ALGORITHM}

The proposed radial velocity estimation algorithm consists of two stages: increasing the accuracy of partial ambiguous velocity estimates and resolving the ambiguity.

The first stage is necessary because the resolution of the Doppler filterbank, which provides preliminary estimates of the Doppler shift, is typically too low to regard these estimates as sufficiently accurate. It is therefore necessary to perform additional, more sophisticated, spectral analysis. To this end, we propose to employ the Capon spectrum estimator. Let

$x_{1}, x_{2}, \ldots, x_{N}$ denote the consecutive samples of the echo signal from an analyzed pulse trail, and let $L \leq \frac{N}{2}$ denote the width of the local analysis window. First, we compute the matrix

$$
\boldsymbol{R}=\sum_{i} \boldsymbol{y}_{i} \boldsymbol{y}_{i}^{H}
$$

where

$$
\boldsymbol{y}_{i}=\left[\begin{array}{c}
x_{i} \\
x_{i+1} \\
\vdots \\
x_{i+L-1}
\end{array}\right]
$$

Second, we search for a local maximum of the Capon spectrum, which is computed from the following formula [4]

$$
S(f)=\frac{1}{\boldsymbol{a}^{H}(f) \boldsymbol{R}^{-1} \boldsymbol{a}(f)},
$$

where $\boldsymbol{a}^{H}(f)=\left[\begin{array}{lllll}1 & e^{j 2 \pi f \cdot P R I} & e^{j 2 \cdot 2 \pi f \cdot P R I} & \ldots & e^{j(L-1) \cdot 2 \pi f \cdot P R I}\end{array}\right]^{T}$, and $T_{P}$ is the current pulse trail's PRI. To implement the search, one may employ a derivative-free numerical optimizer. As a result, one obtains the increased-accuracy Doppler shift estimate $f_{D}$. Should it, however, occur that

$$
f_{D}>\frac{1}{2 T_{P}}
$$

then one should correct this estimate in the following way

$$
f_{D} \leftarrow f_{D}-\frac{1}{T_{P}}
$$

The final step is the computation of the ambiguous radial velocity estimate

$$
v_{R}=\frac{c}{2 F_{C}} f_{D}
$$

where $F_{C}$ denotes the carrier frequency.

By analyzing signals collected from consecutive pulse trails using (4)-(9), one obtains the sequence of pairs $\left(v_{R, 1}, v_{B, 1}\right),\left(v_{R, 2}, v_{B, 2}\right), \ldots,\left(v_{R, K}, v_{B, K}\right)$, where

$$
v_{B, i}=\frac{c}{2 F_{C, i}} \cdot \frac{1}{P R I}
$$

denotes the first blind velocity for the $i$-th pulse trail. These pairs serve as data to the velocity ambiguity resolution step. Formally, the problem of resolving the velocity ambiguity can be stated as follows. Let 


$$
\boldsymbol{A}=\left[\begin{array}{ccccc}
1 & -v_{B, 1} & & & \\
1 & & -v_{B, 2} & & \\
\vdots & & & \ddots & \\
1 & & & & -v_{B, K}
\end{array}\right] \quad \boldsymbol{b}=\left[\begin{array}{c}
v_{R, 1} \\
\vdots \\
v_{R, K}
\end{array}\right]
$$

Then, the available partial estimates satisfy the equation

$$
A x=b+\epsilon,
$$

where the first element of the vector $\boldsymbol{x}$ contains the true target velocity and its remaining elements are integers. The vector $\boldsymbol{\epsilon}$ appearing in the right-hand side of (12) is the vector of random errors. The proposed method of finding a vector $\widehat{\boldsymbol{x}}$ that has approximately the structure of the true vector $\boldsymbol{x}$ and yields good fit to the data is described below.

First, we find the minimum of the following cost function for $n=0,1,2, \ldots, n_{\max }$,

$$
Q(n)=\left\|\boldsymbol{y}_{n}-\operatorname{round}\left(\boldsymbol{y}_{n}\right)\right\|,
$$

where

$$
\boldsymbol{y}_{n}=\boldsymbol{x}_{\mathbf{0}}(2: \text { end })+n \boldsymbol{k}_{\boldsymbol{A}}(2: \text { end }),
$$

$\boldsymbol{w}(2:$ end $)$ denotes the subvector of a vector $\boldsymbol{w}$ that contains all its element except the first one,

$$
\boldsymbol{k}_{\boldsymbol{A}}=\left[\begin{array}{c}
v_{B, 1} \\
1 \\
v_{B, 1} / v_{B, 2} \\
v_{B, 1} / v_{B, 3} \\
\vdots \\
v_{B, 1} / v_{B, K}
\end{array}\right],
$$

and $\boldsymbol{x}_{\mathbf{0}}$ is computed as follows

$$
\begin{aligned}
& \boldsymbol{x}_{\mathbf{0}} \leftarrow \boldsymbol{A}^{\#} \boldsymbol{b} \\
& \boldsymbol{x}_{\mathbf{0}} \leftarrow \boldsymbol{x}_{\mathbf{0}}-\boldsymbol{k}_{\boldsymbol{A}} \boldsymbol{x}_{\mathbf{0}}(2),
\end{aligned}
$$

where $\boldsymbol{A}^{\#}$ is a pseudoinverse of $\boldsymbol{A}$, while $\boldsymbol{x}_{\mathbf{0}}(2)$ is the second element of $\boldsymbol{x}_{\mathbf{0}}$.

Second, after finding the optimal value of $n$, one can obtain the target from the following formula

$$
\hat{v} \leftarrow x_{0}(1)+n \boldsymbol{k}_{A}(1) .
$$

Finally, if the resultant velocity exceeds $v_{\max } / 2$, where $v_{\max }$ denotes the system blind velocity, the following operation should be performed

$$
\hat{v} \leftarrow \hat{v}-v_{\max }
$$

\section{RESULTS OF COMPUTER SIMULATIONS}

Consider an X-band radar $\left(F_{C}=10 \mathrm{GHz}\right)$ that transmits two pulse trails with PRIs of $200 \mu \mathrm{s}$ and $220 \mu$ s. Each pulse trails consists of $N=6$ pulses. The blind velocities in each pulse trails are equal to $75 \mathrm{~m} / \mathrm{s}$ and $68.18 \mathrm{~m} / \mathrm{s}$, respectively. The system blind speed is therefore $750 \mathrm{~m} / \mathrm{s}$, which allows for unambiguous measurement of radial velocities up to 375 $\mathrm{m} / \mathrm{s}$. Suppose that the observed target moves with the velocity equal to $315 \mathrm{~m} / \mathrm{s}$. The Doppler shift frequency of its echo equals $21 \mathrm{kHz}$ [c.f. (1)], and, after aliasing, $1000 \mathrm{~Hz}$ and $-1727 \mathrm{~Hz}$ respectively [c.f. (2)]. Fig. 2. shows typica Capon spectra obtained for $L=3$ and single-pulse signal to noise ratio equal to $20 \mathrm{~dB}$. Observe that, despite the low number of pulses in each trail, the accuracy of the frequency estimates is sufficient to perform the second stage of the algorithm successfully. 
Fig. 3. Shows the histogram of final velocity estimates obtained from 1000 Monte Carlo experiments. Even though the proposed velocity resolution method is not optimal, its accuracy is absolutely satisfactory from the practical standpoint in the scenario presented here, the root mean squared error of the final velocity estimates is only $0.31 \mathrm{~m} / \mathrm{s}$.
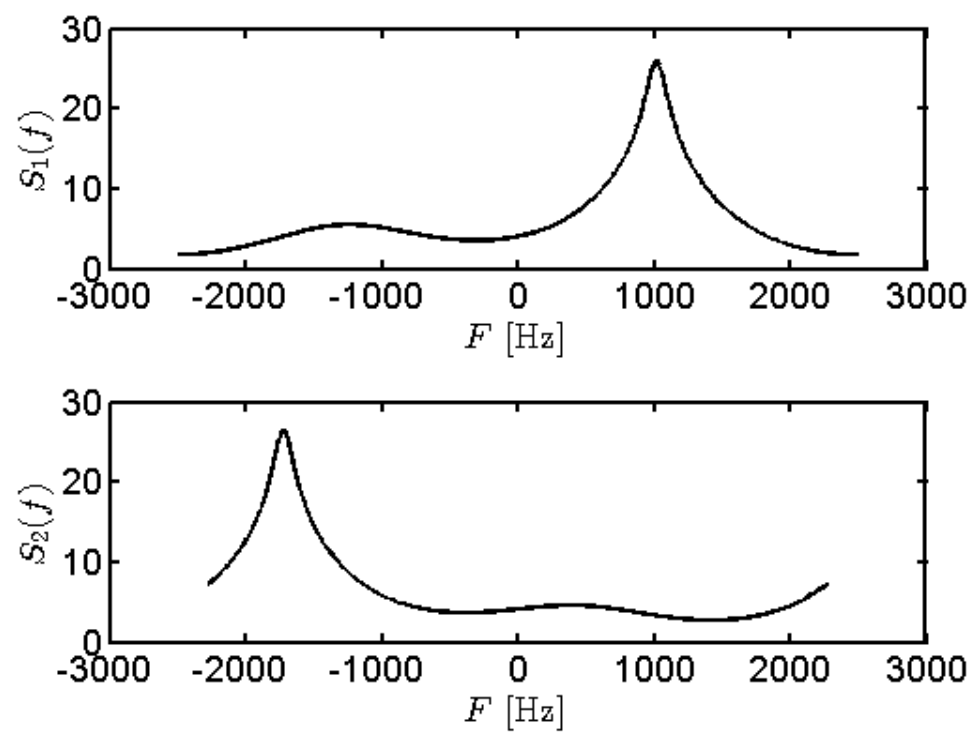

Fig. 2. Typical Capon spectra obtained in the considered simulation experiment.

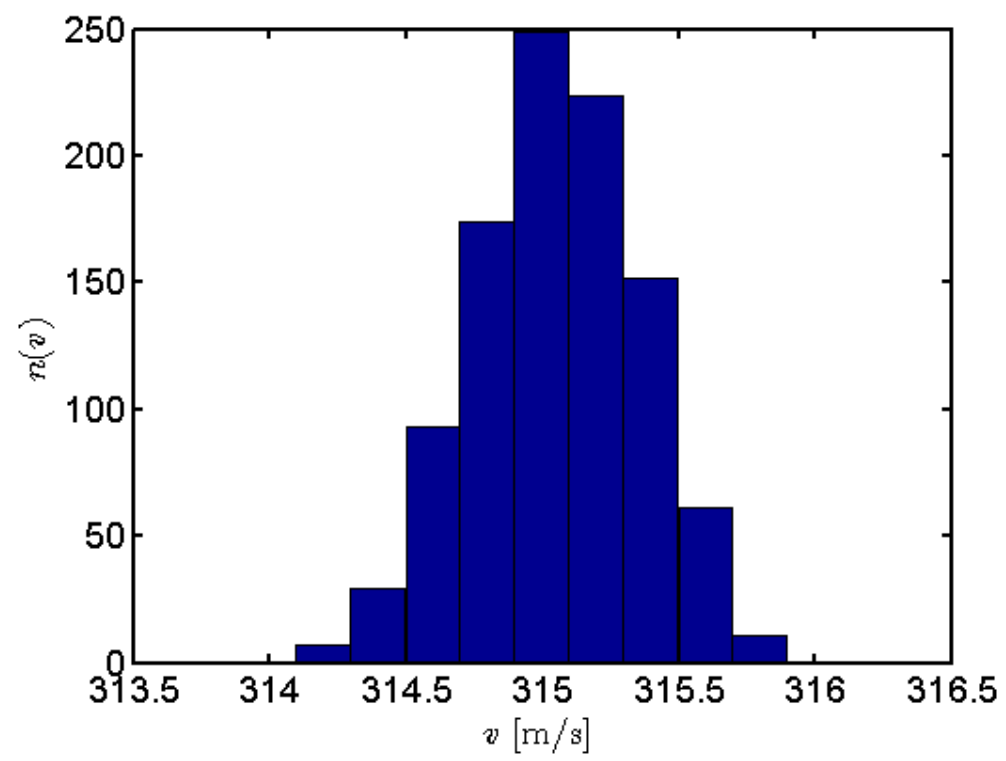

Fig. 3. Histogram of velocity estimates obtained in 1000 Monte Carlo trials. 


\section{CONCLUSIONS}

We proposed the concept of low-cost software extension of radar systems designed to work in the low-PRF mode. The proposed modification equips such systems with the capabilities to estimate the radial velocity and to resolve the range ambiguity occurring for detections of targets that are outside the system's instrumented range. We described the proposed velocity estimation algorithm in details and verified its performance using computer simulations. The results indicate that its accuracy is sufficient for practical applications. Detailed discussion of the issue of range ambiguity is discussed in a separate paper.

\section{REFERENCES}

[1] Barton, D. K., [Radar System Analysis and Modeling], Artech House, Norwood (2005).

[2] Stawiarski, K. and Meller, M. "Adaptation of radar software to work with ambiguous distance measurement," Proc. 2019 Radioelectronic Systems Conference, Jachranka, Poland (2019).

[3] Skolnik, M. [Radar Handbook], McGraw-Hill, New York (2008).

[4] Capon, J. "High resolution frequency-wavenumber spectrum analysis," Proceedings of the IEEE 57, 1408-1418 (1969). 